
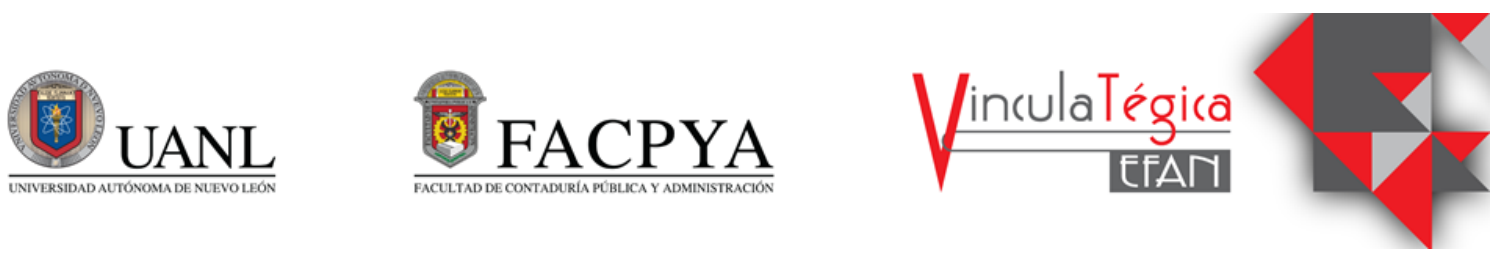

\title{
Influencia de las motivaciones hedónicas en la intención de compra de una vivienda en el Área Metropolitana de Monterrey
}

\author{
Karen Viridiana Segovia Martínez ${ }^{1}$ \\ ${ }^{1}$ Universidad Autónoma de Nuevo León, Facultad de Arquitectura, viridiana_12_2@hotmail.com, Av, Pedro de \\ Alba s/n, Ciudad Universitaria, 66455 San Nicolás de los Garza, N.L. 8183294160
}

Información del artículo revisado por pares

Fecha de aceptación: junio-2021

Fecha de publicación en línea: diciembre-2021

DOI: https://doi.org/10.29105/vtga7.1-123

\section{Resumen}

En el presente artículo se expone una reseña histórica y teórica para comprender los conceptos básicos sociales en el área metropolitana de Monterrey y su desarrollo social, económico e ideológico, así como la relación que tiene con el resto del mundo. El objetivo de esta investigación será comprobar si realmente es necesario incluir un estudio social profundo para el diseño de las viviendas prototipo en el Área Metropolitana de Monterrey (AMM) en resaltar las características de las personas que habitan en la región de estudio y saber que buscan al momento de comprar una vivienda, utilizamos métodos cuantitativos en la investigación para ello se realizó un breve cuestionario de 18 preguntas a 100 personas que han realizado alguna remodelación y han requerido servicios de diseño y construcción. Analizamos en esta investigación las características hedónicas en cuanto a los espacios de la casa habitación, nivel de ingreso en conjunto de la vivienda, descubrir los espacios de mayor interés de los individuos y ver que tan conformes están con los espacios sugeridos en las viviendas ofrecidas al público en serie en la actualidad. Los principales hallazgos son que los individuos que tienen una casa en seria son los que mas han remodelado su casa y tienen mas preferencia por espacios libres no delimitados, los espacios de área exterior libre y cocina los prefieren mas que la sala y comedor, también que es necesario reestructurar los espacios, poner más atención a las necesidades de las personas que habitamos en este tiempo y con nuestras costumbres.

Palabras clave: espacios, Monterrey, hedónico, diseño, vivienda.

\begin{abstract}
This article presents a historical and theoretical review to understand the basic social concepts in the Metropolitan Area of Monterrey and its social, economic, and ideological development, as well as the relationship it has with the rest of the world. The objective of this research will be to verify if it is necessary to include a deep social study for the design of prototype homes in the Metropolitan Area of Monterrey (AMM) to highlight the characteristics of the people who live in the AMM and to know what they are looking for when buying a home, we used quantitative methods in the research for this, a short questionnaire of 18 questions was made to 100 people who have carried out some remodeling and they have required design and construction services. In this research we analyze the hedonic characteristics in terms of the spaces of the house, the income level of the house as a whole, discover the spaces of greatest interest to the individuals and see how they are in agreement with the spaces suggested in the homes offered to the serial audience today. The main findings
\end{abstract}


are that the individuals who owns a serial house, mostly, are the ones who have remodeled their house and have a greater preference for open spaces that are not delimited, the free outdoor area and kitchen spaces are preferred more than the living room and dining room areas, also that It is necessary to restructure the

\section{INTRODUCCIÓN}

El aporte de esta investigación será comprobar si realmente es necesario incluir un estudio social profundo para el diseño de las viviendas prototipo en el Área Metropolitana de Monterrey (AMM).

En el AMM existen 5,784,442 habitantes y 1,655,690 viviendas particulares habitadas según el INEGI 2020, para que los usuarios desarrollen su vida cotidiana de manera satisfactoria es necesario que estas viviendas cumplan con sus necesidades, existen 2 teorías hablando de las necesidades humanas (universalistas), sostiene que las necesidades básicas de todos los seres humanos son fundamentalmente las mismas $\mathrm{y}$ (relativistas) que intentan negar la noción de las necesidades humanas como universales, determinando que las necesidades son distintas en función del sexo, edad, raza, cultura, normas sociales adquiridas y percepción individual (Puig, 2012).

La ciudad de Monterrey entrelaza las estructuras industriales con los procesos de formación de una burguesía local, conservadora y resistente a las fuerzas centralizadoras del Estado mexicano (Smith,2008). En el diseño de los espacios de las viviendas esto es muy evidente ya que la distribución y las áreas se repiten sin tomar en cuenta nuestro modelo actual de familia y simplemente se replica, sería de mucha utilidad si existiera un análisis conocer las áreas de las que spaces, to pay more attention to the needs of the people who live in this time and with our customs.

Keywords: spaces, Monterrey, hedonic, design, living place.

JEL: D13, I31, L74.

se puede prescindir (en caso de contar con espacio limitado) para mejorar la calidad de vida, con este cambio podríamos tener como resultado más comodidad para el usuario que al final se traduciría en seguridad, progreso y bienestar.

Con el paso del tiempo ha sido notorio el incremento de la inseguridad, podríamos suponer que gran cantidad de los fraccionamientos en serie se hacen sin un correcto estudio de los espacios, los niños y jóvenes salen de sus viviendas para tener mayor comodidad y en medio del ocio y estudio es que comienzan a perder la guía por parte de sus familias. La globalización ha traído como consecuencia que los neoloneses están cambiando su estilo de vida, pensamientos y la estructura familiar, estos cambios provocarán que se vivan diferente los espacios como cocina, comedor, sala, etcétera. Incluso hoy en día las actividades ligadas al trabajo están muy inmersas en el ámbito doméstico Por ello es necesario replantearnos qué tan eficientes son los espacios con los que contamos en las viviendas que actualmente se ofrecen.

El año 2020 en el que entró el COVID-19, nuestro estilo de vida cambio con radicalidad, todas las personas se han visto en la necesidad de realizar todas las actividades en su casa, destacó que los espacios no contaban con la flexibilidad 
de diseño necesaria para adaptar todas estas actividades en un solo espacio y esto ha provocado que sea muy complicado el confinamiento que se requiere.

La cultura se ha nutrido a su vez de nuevos elementos como lo son el conjunto de símbolos, valores, habilidades, conocimientos, significados, formas de comunicación y organización social, entre otros, que amplían notoriamente lo que hoy entendemos como tal (Sánchez, 2006). Tomando en cuenta que esta zona geográfica se apega fuertemente a sus costumbres existe la posibilidad que los espacios se sigan diseñando de una manera en la que actualmente no se explote al máximo, ya que las personas de hoy no viven la realidad que se vivió en años anteriores, socialmente cambiamos y evolucionamos.

Se define la vivienda como solución de diseño en un enfoque amplio que involucra la evaluación del espacio habitable a escala arquitectónica, la relación con su entorno inmediato y con la ciudad a escala urbana (Pérez, 2011). En el documento de Pérez se muestra una tabla en la que se explica cómo las posibilidades económicas definen las tradiciones, gustos, costumbres, preferencias y expectativas del usuario y cómo se relaciona fuertemente el pasado con las tradiciones, el presente con los gustos, preferencias y costumbres; y el futuro con las expectativas.

El objetivo de este artículo es encontrar la manera en que podamos aportar un nuevo enfoque de diseño y basándonos en las características hedónicas del usuario sean más sencillas las ventas, optimizar los espacios, conocer el entorno que viven los habitantes del AMM y ofrecer una buena calidad de vida con espacios altamente funcionales para cualquier actividad deseada por el usuario a través de su vida en años futuros.

Esta investigación pretende beneficiar a futuras generaciones de fraccionadores, diseñadores y usuarios finales resaltando las motivaciones hedónicas al momento de la compra de una vivienda, estas consideraciones podrán ser tomadas en cuenta para una mejor inversión de recursos, ahorro en adecuaciones y una mejor calidad de vida.

\section{MARCO TEÓRICO}

En las grandes ciudades alemanas ocurrieron hechos transcendentales en el siglo XX entre la vivienda social y la arquitectura contemporánea, No es por gusto que la arquitectura dejara de construir palacios y catedrales, sino que las transformaciones vividas en el pasado siglo rediseñaron radicalmente la relación entre arquitectura y sociedad. Este cambio de escala ha llevado a la gran arquitectura a preocuparse también por la vivienda social, en un gesto en parte democratizador que ha permitido al gran público a acceder a una vivienda «firmada» (López, 2003). El antecedente de estas prácticas es que realmente antes de ese siglo a la gente que no pertenecía a la alta sociedad se le brindaba un espacio óptimo en forma rectangular, no se consideraba que se requiriera un diseño o una 
distribución especiales, se esperaba que el usuario supiera administrar el poco dinero que tenía y simplemente cumplir con la necesidad de tener un techo.

En Argentina por los años 1948 y 1949 los barrios correspondían fielmente al modelo de barrio vecinal donde cada casa individual era habitada por una familia, logrando una relativa independencia de sus vecinos remitía claramente al modelo del barrio obrero centroeuropeo construido en bloques de viviendas. Compartían los accesos, circulaciones y espacios exteriores de la planta baja, circunstancias que favorecían el intercambio social entre los vecinos (Aboy, 2003). En este punto vemos como la necesidad y las costumbres de las personas van cambiando y es necesario actuar en función a ellas. Hoy en día necesitamos más que habitar una casa, pedimos confort, espacios funcionales, agradables a la vista y que cumplan con las necesidades generales de las personas que viven en la época actual.

Con la aparición de nuevas formas familiares: individuos solos, parejas, ancianos, estudiantes, grupos genéricos o profesionales, se incorporan a la vivienda nuevas actividades, dando un renovado protagonismo y sentido a los distintos espacios que la conforman (Fisch, 2011). Es necesario encontrar la manera en que cada familia pueda desarrollarse de manera óptima sean cuales sean sus actividades y gustos, que todo sea adaptable a nuevos pasatiempos, empleos y maneras de recreación, más allá de llegar y dormir a las casas buscamos un espacio en el que podamos trabajar, hacer ejercicio, convivir con familia y amigos, compartir una comida, ver la tv, entre otras cosas.

Más allá de los cambios sociales está el ciclo de las personas que habitan una vivienda y con esta nos referimos a las etapas de la vida de un ser humano niñez, adolescencia, adultez y tercera edad, en cada etapa las personas tenemos distintas necesidades y de ahí viene la importancia del análisis de los espacios y ser conscientes de que tan valioso puede ser la flexibilidad de espacios. Los edificios no son jamás definitivos. No pueden ser permanentes cuando la sociedad que los rodea está en plena evolución (González, 2008).

Respecto de la vivienda, durante el siglo $\mathrm{XX}$ se reciclan dos inventos tipológicos importantes en el mundo del habitar; uno es el atelier, espacio de trabajo de los artistas, rescatado por Charles-Édouard Jeanneret-Grisen "Le Corbusier" en los años 20, que se constituye en expresión de la vivienda moderna y dio lugar a gran cantidad de experimentación en vivienda durante todo el siglo. El tipo tiene un espacio determinado: un volumen de doble altura y una entreplanta; está permitía a lo largo del tiempo diferentes propuestas. El segundo es el loft, usado también por artistas, que se origina en Nueva York, a fines de los 60, en el mismo período en que se origina la práctica de la rehabilitación como parte del campo de la arquitectura (Fisch, 2011). A grandes rasgos podríamos considerar que una vivienda con estos dos estilos arquitectónicos sería muy funcional teóricamente 
ya que estos te ofrecen la opción a un cambio según las etapas, cambios y evoluciones que puedan existir en una familia.

$\mathrm{Al}$ tener en cuenta al hombre como usuario y generador de actividades que son, a su vez, responsables de la forma y dimensión de los espacios arquitectónicos, podemos saber cuáles son los espacios arquitectónicos, podemos saber cuáles son los espacios mínimos que el hombre necesita para desenvolverse diariamente (Fonseca, 2012). Zona social, zona privada y

\section{MÉTODO}

Gracias a un muestreo no probabilístico que realizamos obtuvimos una base de datos usando la plataforma de Google para recolectar la información requerida para nuestro muestreo de la que nos beneficiamos realizando un análisis de estadística descriptiva e inferencial con estadísticos descriptivos (media, varianza y desviación estándar), estadísticos de frecuencia (indican el número de veces que se repiten las respuestas).

Utilizamos el programa SPSS para obtener los resultados de las tablas 1, 2, 3 y 4, que nos zona de servicio son zonas que cumplen sus funciones por medio de las áreas sala, comedor, cocina, recamaras, baños, cocina, lavandería, entre otras, para que tengan un correcto funcionamiento debemos tomar en cuenta las medidas ergonométricas que ya han sido estudiadas a través del tiempo y hacer una comparación del espacio con el que contamos, el gusto del usuario en cuanto al estilo de los muebles, estructura familiar y las edades con las que cuentan los usuarios de la vivienda.

ayudó a determinar los resultados de una manera más acertada. A continuación, presentamos la tabla 1 en la que aparece la descripción de las 18 variables que consideramos, en el lado izquierdo tiene el nombre clave de cada variable y en el lado derecho se lee la descripción de cada una. En las primeras 4 variables recolectamos datos de básicos de las personas, de la 5 a la 11 cuestionamos con respecto a características generales de ingreso y características de la vivienda en donde habitan y de la 12 a la 18 se preguntó con respecto a las personas que viven la casa y la importancia que le dan a ciertos espacios basándonos en Moreno y Alvarado (2011)

\begin{tabular}{ll}
\hline \multicolumn{1}{c}{ Concepto } & \multicolumn{1}{c}{ Tabla 1. Descripción de las variables } \\
\hline Género & \multicolumn{1}{c}{ Descripción } \\
Escolaridad & Género del encuestado \\
Edad & Grado académico obtenido \\
Civil & Años del encuestado \\
Ingreso & Condición Social \\
Pertenencia & Ingreso monetario mensual de vivienda \\
Inmueble & Propiedad del inmueble \\
Terreno & Tipo de inmueble donde reside \\
& M2 de terreno de vivienda
\end{tabular}




\begin{tabular}{ll} 
Municipio & Zona de residencia en AMM \\
Remodelación & Se ha remodelado la vivienda \\
Precio & Que tan importante es el precio al adquirir una vivienda \\
Habitantes & Cuántos habitantes viven en la vivienda \\
Reuniones & Cuantas personas socialmente se reciben en la vivienda \\
Sala & Que tan importante es el espacio de sala \\
Comedor & Que tan importante es el espacio de comedor \\
Cocina & Que tan importante es el espacio de cocina \\
Área exterior libre & Que tan importante es el espacio de área exterior libre \\
Espacios & Que tan importante es esta área \\
\hline
\end{tabular}

Haciendo uso de las variables que hemos descrito anteriormente que se tomaron de la encuesta realizada iniciaremos la interpretación de los resultados que nos arrojó la tabla 2. Resalta que de las personas encuestadas el género femenino fue predominante con respecto al de género masculino debido a la media 1.60 , siendo 1 hombre y 2 mujer. La edad promedio de estos se encuentra entre los 35 y 36 años. con un nivel de estudios de licenciatura. De acuerdo con la media reflejada en estado civil podemos inferir que predomina la gente que vive en unión libre.

\begin{tabular}{lcccc}
\hline \multicolumn{5}{c}{ Tabla 2. Variables de control } \\
\hline Variable & Mínimo & Máximo & Media & Desv. Desviación \\
\hline Escolaridad & 1 & 6 & 3.18 & .916 \\
Edad & 26 & 66 & 35.51 & 10.295 \\
Género & 1 & 2 & 1.60 & .493 \\
Ingreso & 1 & 4 & 2.55 & 1.077 \\
Civil & 1 & 4 & 2.46 & .886 \\
\hline
\end{tabular}

Fuente: elaboración propia.

\begin{tabular}{ccccc}
\hline \multicolumn{4}{c}{ Tabla 3. Variables de control especificas } \\
\hline & Mínimo & Máximo & Media & Desv. Desviación \\
\hline $\begin{array}{c}\text { ¿Qué tan importante es el espacio } \\
\text { designado para la sala? }\end{array}$ & 1 & 3 & 1.61 & .632 \\
$\begin{array}{c}\text { ¿Qué tan importante es el espacio } \\
\text { designado para el comedor? }\end{array}$ & 1 & 3 & 1.79 & .683
\end{tabular}


¿Qué tan importante es el espacio designado para la cocina?

¿Qué tan importante es el espacio designado para el área exterior libre?

¿Qué tipo de espacios prefiere en

$\begin{array}{llll}1 & 3 & 1.50 & .610 \\ 1 & 4 & 1.48 & .731 \\ 1 & 2 & 1.76 & .426\end{array}$

La interpretación de los resultados que nos arrojó la tabla 3 la cual se conforma de resultados de las preguntas que se realizaron para poder conocer cuál es el orden hedónico del usuario en cuanto a preferencia de espacios.

(Se aplicaron nomenclaturas del 1 al 6 en la variable de escolaridad, siendo 1.-carrera Fuente: elaboración propia.

Donde los encuestados toman como prioridad los espacios en el siguiente orden: área social exterior libre, cocina, sala, comedor. Para la valoración de preferencia en espacios delimitados por área y espacios libres en una vivienda. técnica, 2.-preparatoria, 3.-licenciatura, 4.maestría, 5.-doctorado, 6.- sin estudios, también a la variable de ingreso se le denomino con la nomenclatura del 1 al 4, 1.-menos de $\$ 15,000.00 \mathrm{mx}, \quad 2$. -de $\$ 15,000.00 \mathrm{mx} \quad \mathrm{a}$ $\$ 30,000.00 \mathrm{mx}, \quad 3 .-$ de $\$ 30,000.00 \mathrm{mx}$ a $\$ 60,000.00 \mathrm{mx}, 4 .-\$ 60,000.00 \mathrm{mx}$ o más).
prefiere en casa? Siendo que la nomenclatura es 1.- Espacio delimitado por área y 2.- Espacios libres, la media nos arrojó que más del 70\% de los encuestados prefieren áreas libres y no delimitadas por espacio. 
En la tabla 4 representamos las respuestas de los encuestados con frecuencias y porcentajes de preguntas acerca de la vivienda y quien habita en ella.

\begin{tabular}{|c|c|c|}
\hline \multicolumn{3}{|c|}{ Tabla 4. Características generales de los encuestados } \\
\hline Variable & Frecuencia & Porcentaje \\
\hline \multicolumn{3}{|c|}{ Pertenencia } \\
\hline Propia & 78 & 77.2 \\
\hline Rentada & 13 & 12.9 \\
\hline Prestada & 10 & 9.9 \\
\hline Total & 101 & 100 \\
\hline \multicolumn{3}{|c|}{ Vivienda } \\
\hline Casa prototipo & 62 & 61.4 \\
\hline Casa con diseño exclusivo & 35 & 34.7 \\
\hline Departamento & 4 & 4 \\
\hline Total & 101 & 100 \\
\hline \multicolumn{3}{|c|}{ Remodelación } \\
\hline Sí & 57 & 56.4 \\
\hline No & 26 & 25.7 \\
\hline No, pero me gustaría & 18 & 17.8 \\
\hline Total & 101 & 100 \\
\hline \multicolumn{3}{|c|}{ Habitantes } \\
\hline 1 a 4 habitantes & 80 & 79.2 \\
\hline 4 a 8 habitantes & 18 & 17.8 \\
\hline 8 o más habitantes & 3 & 3 \\
\hline Total & 101 & 100 \\
\hline \multicolumn{3}{|c|}{ Reuniones } \\
\hline 901 invitadn & 4 & 4 \\
\hline Fuente: Elaboración propia. & 30 & 29.7 \\
\hline 8 a 15 invitados & 40 & 39.6 \\
\hline 15 o mas & 27 & 26.7 \\
\hline Total & 101 & 100 \\
\hline \multicolumn{3}{|c|}{ Espacios } \\
\hline Espacios delimitados por área & 24 & 23.8 \\
\hline Espacios libres & 77 & 76.2 \\
\hline Total & 101 & 100 \\
\hline
\end{tabular}




\section{RESULTADOS}

En base a las variables de control, específicas y las características generales del encuestado obtenidos en la investigación de método no probabilístico (encuesta).

Grafica 1. Tipo de vivienda según encuestados

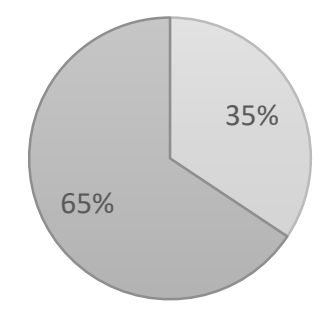

$\square$ Diseño exclusivo $\square$ Diseño prototipo

Tenemos como resultado que en el muestreo que se realizó en el AMM hay más individuos con viviendas de diseño prototipo el cual es el $65 \%$ y solo el 35\% de los 100 encuestados tienen una vivienda con diseño exclusivo.

Grafica2. Tipo de vivienda e ingreso monetario mensual según encuestados

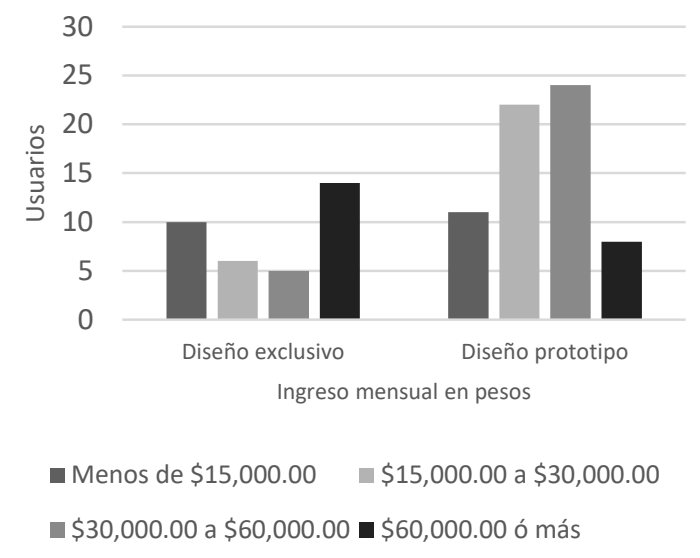

De acuerdo con la grafica 2 podemos apreciar que los usuarios con diseño exclusivo en sus viviendas cuentan con un ingreso mensual mas elevado que los usuarios de vivienda con diseño prototipo

Grafica 3. Tipo de vivienda según encuestados 


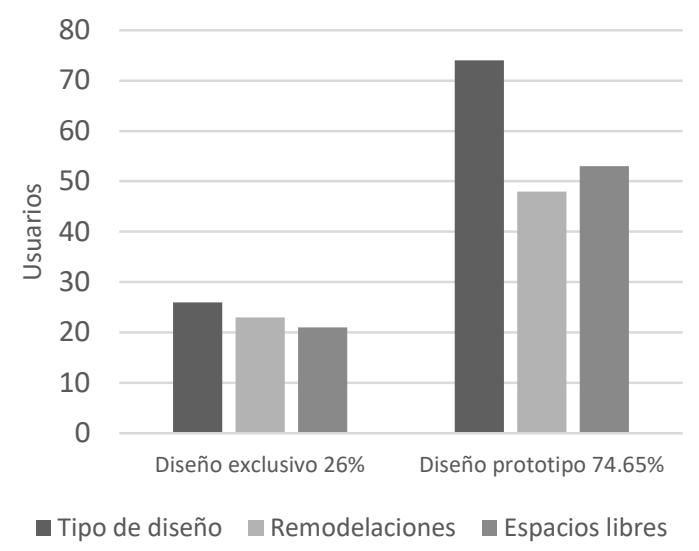

En la grafica 3, nos podemos percatar de la significancia respecto a diseño exclusivo y prototipo, la mayoría de los usuarios cuentan con una vivienda prototipo y son los que mas han remodelado su vivienda y los que prefieren espacios libres y no delimitados.

Grafica 4. Escala de importancia hedónica, según usuario AMM

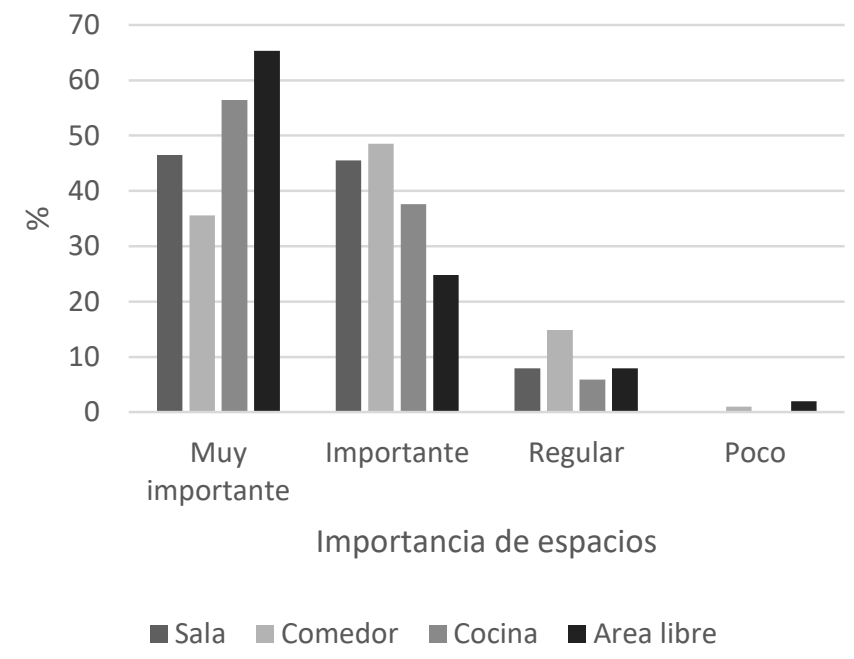

En la gráfica 4, se ha visto una preferencia muy acentuada por los espacios de área exterior libre y cocina vs sala y comedor.

\section{CONCLUSIONES}

Después de analizar los resultados que arrojaron las encuestas pudimos encontrar que hay más individuos con casas en serie, que los individuos que tienen una casa en serie son los que más han remodelado su casa y tienen más preferencia por espacios libres no delimitados, los espacios de área exterior libre y cocina los prefieren más que la sala y comedor 
Según la investigación el concepto de propiedad privada para los individuos de clase media es relativamente nuevo (siglo 20) y ha habido un gran avance desde entonces, creemos valioso que esta evolución sea más rápida y con un estudio adecuado ya que el punto número uno para tener una construcción exitosa y funcional son las ingenierías y la planeación.

Tomando en cuenta las definiciones, marco teórico y nuestros resultados podemos destacar que es necesario reestructurar los espacios, ser más abiertos en cuanto al diseño y poner más atención a las necesidades de las personas que habitamos en este tiempo y con nuestras costumbres

Una de las limitaciones de este trabajo fue el tamaño de la muestra (100 personas que han solicitado el trabajo de arquitectos o ingenieros para la ejecución de sus remodelaciones. Este proyecto es un buen punto de partida para el diseño de futuras metodologías que ayude a ampliar el análisis en el tema del desarrollo de viviendas y los efectos sociales que pueden partir de las mismas. 


\section{REFERENCIAS}

Aboy. Rosa., (2003) La vivienda social en buenos aires en la segunda posguerra, Scripta Nova vol. VII, 146 en http://www.ub.edu/geocrit/sn/sn-146(031).htm

Fisch. Sara., Etulain. Juan Carlos., Pagani. Gustavo., (2011). Las problemáticas conceptuales para el diseño de la vivienda contemporánea. Cuaderno urbano. Espacio, cultura, sociedad. Vol. 11 PP. 27-57, en https://www.redalyc.org/pdf/3692/369236772002.pdf

Fonseca, Xavier. Las medidas de una casa, Pax México. En: https://books.google.es/books?hl=es\&lr=\&id=_17MSFgDc1wC\&oi=fnd \&pg=PA7\&dq=espacios+d e+servicio+casa\&ots=YkJDyzjJLH\&sig=WepuRgIIQRih9lnbFkme35 rbnM\#v=onepage \&q=espac ios $\% 20 \mathrm{de} \% 20$ servicio\%20casa\&f=false

González. Xavier (2008) at+architecture publishers, en: https://aplust.net/blog/flexible_para_sobrevivir/idioma/es/

López. Díaz. Jesús., (2003) La relevancia de la vivienda social en el origen de la arquitectura contemporánea, UNED. Espacio, Tiempo y forma, serie VII. 179-197 en http://espacio.uned.es/fez/eserv/bibliuned:ETFSerie7-B935FDE5-97CB-26D6-95D496BB55580539/Documento.pdf

Moreno, R. y Alvarado, E. (2011). El entorno social y su impacto en el precio de la vivienda: un análisis de precios hedónicos en el área metropolitana de Monterrey. Trayectorias, 14(33), 131-147.

Pérez. Pérez. Alex Leandro (2011) Bases para la evaluación del diseño de la vivienda arquitectura y urbanismo. Vol. XXXII pp. 30-35 en https://www.redalyc.org/pdf/3768/376839862007.pdf

Puig, Llobet. Montserrat., Sabater. Mateu, Pilar., Rodríguez. Ávila. Nuria., (2012) Evolución del concepto según la perspectiva social. Aposta. Revista de ciencias sociales. PP. 1-12, en https://www.redalyc.org/pdf/4959/495950250005.pdf

Sánchez. Suárez. Aurelio., (2006). La casa maya contemporánea. Usos, costumbres y configuración espacial, $\quad$ Península $\quad$ ISSN $1870-5766$ en http://www.scielo.org.mx/scielo.php?script=sci_arttext\&pid=S1870-57662006000200003

Smith. Pussetto. Cintia., García. Vázquez. Nancy Janett., Pérez. Esparza. Jesús David., (2008) Análisis de la ideología empresarial regiomontana. Un acercamiento a partir del periódico El Norte. http://www.scielo.org.mx/pdf/confines/v4n7/v4n7a1.pdf 